\title{
Resúmenes
}

\section{Tesis de Maestría de la Escuela Graduada de Trabajo Social Beatriz Lassalle 2020 - 2021}

Jannette Rodríguez Ramírez

Compiladora

Universidad de Puerto Rico

jannette.rodriguez3@upr.edu https://orcid.org/0000-0001-5816-7629

Cómo citar este artículo (estilo APA) / How to cite this article (APA style) Rodríguez-Ramírez, J. (2021). Resúmenes de tesis de maestría presentadas en mayo 2020. Análisis, 17(1), 1-4.

https://doi.org/10.54114/revanlisis.v17i1.19418

Derechos de autoria / Copyright: @ 2021 Rodriguez-Ramirez, Jannette

Este es un artículo de acceso abierto y distribuido bajo los términos de la licencia y políticas de Creative Commons

Attribution 4.0 International License 
$2020 \approx$

Berríos, Miosotis Del Mar; Reynoso, Ana M. \& Martínez, Naomi M. (2020). La vida de la madre estudiante universitaria: Aspectos sociales, económicos y académicos en estudiantes de la Facultad de Ciencias Sociales de la Universidad de Puerto Rico en Río Piedras hasta mayo 2020.

Resumen

Esta investigación presenta los diversos problemas que experimenta la población de madres estudiantes universitarias y cómo inciden en su cotidianidad. La metodología implementada fue una con enfoque cualitativo, y alcance exploratorio. Las teorías aplicadas fueron la Teoría Sistémica, la Perspectiva Feminista y el Construccionismo Social. Participaron 10 madres estudiantes universitarias a través de una entrevista semiestructurada. Los hallazgos principales fueron identificados a través de las siguientes categorías: falta de espacios, espacio institucional, relaciones interpersonales y espacios sociales, tiempo de ocio y sentimiento de culpa, prejuicios y limitaciones, roles de género y sobrecargas, prejuicios internalizados, reto económico, importancia del apoyo, la oferta académica en la Universidad de Puerto Rico en Río Piedras, los retos de ser madre estudiante, y, la distribución del tiempo. De los hallazgos se evidenció que existe una carencia de recursos en el área social, económica y académica dirigidos a esta población.

Doelter Morales, Fransheska M. \& Martínez Miranda, Jonathan E. (2020). Hongos mágicos... más allá de las alucinaciones: Análisis político-social y estudio exploratorio del conocimiento y las percepciones que tiene la comunidad de la Universidad de Puerto Rico, Recinto de Río Piedras, sobre el uso de los hongos psicodélicos a diciembre de 2020.

\section{Resumen}

En esta investigación exploramos el conocimiento, las percepciones y los discursos que tienen las personas en la comunidad de la UPR, Recinto de Río Piedras, y comunidades aledañas, sobre los hongos psicodélicos. Presentamos la crisis de salud mental en Puerto Rico y los servicios que se ofrecen para manejarla. Proponemos mirar otras alternativas a los tratamientos de salud mental existentes, que consisten en el consumo farmacológico como único método, ya que está muy atrasado en cuanto al bienestar que ofrecen a las personas. Por tal razón, exploramos el tema de los hongos psicodélicos, organismo natural que contiene una sustancia llamada psilocibina, que se utiliza en investigaciones clínicas concernientes a la salud mental. Problematizamos la invisibilidad actual de los hongos psicodélicos en Puerto Rico. La investigación fue estructurada siguiendo un diseño metodológico mixto. Como marco conceptual utilizamos la Teoría del Estigma. La recopilación de la información se dio a través de Google Forms. Los resultados son 
alentadores por la cantidad de personas que participaron en la misma y la información que brindaron. Les participantes mostraron conocimiento acerca de los hongos psicodélicos. Las percepciones fueron diversas, y en su mayoría fueron positivas al uso de estos. Existen varios discursos, más allá del discurso terapéutico y de salud mental. La recreación, la cultura, la espiritualidad y la religión, son temas que surgen de esta investigación. Mediante nuestro estudio se abre la puerta, a no tan solo la aceptación de las personas a investigaciones sobre el consumo, la despenalización, descriminalización y legalidad del uso de algunas sustancias, sino que se da apertura para hacer estudios desde distintas disciplinas, como el Trabajo Social. Los hongos psicodélicos podrían ser solución para muchos problemas más allá de la salud mental. Les invitamos a observar esta tesis desde la defensa de los derechos humanos, la apertura a la diversidad y a la interseccionalidad. También, a mirar más allá de las aulas a las que estamos acostumbrados por culpa de un coloniaje, que se ha dedicado a borrar y esconder la memoria colectiva de muchas personas, que no caben en la agenda neoliberal de quienes nos gobiernan.

\section{Fernández, Carlos M.; Mejias, Dalitza \& Rosario, Ambar A. (2020). Percepciones sobre el burnout: Estudiantes de escuelas graduadas de ciencias de la conducta en la Universidad de Puerto Rico, Recinto de Río Piedras, hasta mayo 2020.}

Resumen

Este estudio no experimental-transaccional, se llevó a cabo entre estudiantes de las escuelas graduadas de ciencias de la conducta de la Universidad de Puerto Rico, Recinto de Río Piedras, por medio de un cuestionario en Google Forms. Su propósito fue conocer las percepciones que tienen acerca de los factores sociales, familiares y académicos; la condición económica y política actual del país; las políticas impuestas por la Junta de Control Fiscal a la Universidad, y los factores culturales que contribuyen al burnout. El estudio creó conciencia del burnout en dicha población, debido a que la mayoría de los estudios se enfocan en estudiantes del área de la salud. Se concluye que existen diversas realidades en la comunidad. Sin embargo, el factor económico es importante en el análisis del burnout, ya que es un estresor y provoca la inserción de la estudiante al campo laboral para cumplir con varios roles. 
Lozano, Karolin; Lugo, Samuel A. \& Rodríguez, Glory M. (2020). La construcción de masculinidades desde el punto: Emociones, manifestaciones de violencia y respuesta del Estado en hombres de 21 a 45 años de edad relacionadas al trasiego de drogas en Puerto Rico.

Resumen

En esta investigación se trabajan los temas de masculinidad, trasiego de drogas y el Estado en el contexto de Puerto Rico. Se construye un análisis basándose en las experiencias de los participantes mediante la revisión de literatura en áreas como: la expresión o el manejo de emociones, la respuesta del Estado, y las manifestaciones de violencias. Se expone cómo el Estado, a través de las políticas públicas para atender el problema del trasiego de drogas, manifiesta una masculinidad hegemónica con medidas punitivas y violentas. Y se presenta cómo esto influye e incentiva a que se asuman características de esta masculinidad dentro del trasiego de drogas para poder subsistir. Estos actos violentos se normalizan como forma de sobrevivencia en el sistema y en las instituciones que no responden a las necesidades de las comunidades marginalizadas y vulnerables. Por ende, cientos de jóvenes que están desprovistos de acceso a servicios esenciales para el desarrollo humano y social, se ven impulsados a insertarse en el trasiego de drogas.

\section{Martínez Peña, Delgado Rodríguez, Jackeline \& Valenzuela Encarnación Y. (2020). Un diagnóstico de VIH/SIDA en plena juventud: experiencias de vida, estrategias de afrontamiento y acceso a servicios.}

Resumen

Esta investigación tuvo como propósito conocer las experiencias de vida, estrategias de afrontamiento y el acceso a los servicios para jóvenes de 21 a 34 años, que viven con $\mathrm{VIH} / \mathrm{SIDA}$ en Puerto Rico. Los objetivos fueron: conocer las experiencias de vida y emociones de los jóvenes con $\mathrm{VIH}$, identificar sus estrategias de afrontamiento y apoyo social, y explorar si tienen acceso a los servicios necesarios. Se tomó en cuenta como criterio de participación, estar entre las edades de 21 a 34 años, tener un diagnóstico de VIH/SIDA y residir en Puerto Rico. El diseño de la investigación se realizó desde la metodología cualitativa con carácter descriptivo. La obtención de información se realizó en tres secciones; (a) se recopilaron de datos sociodemográficos con un formulario, que fue analizado desde el aspecto cuantitativo; (b) se implementó una escala de estigma; y (c) se llevó a cabo una entrevista profunda de modo cualitativo con preguntas abiertas, que permitió que se abordara el tema de forma más detallada. La muestra fue recogida por disponibilidad y se entrevistaron a siete personas. Entre los hallazgos más relevantes de la investigación, se encontró que el discrimen y el estigma hacia la población con VIH/SIDA, siguen vigentes en la sociedad $y$, a través, de los profesionales que brindan el servicio. Los y las jóvenes tienen miedo de revelar su diagnóstico a sus familiares y amistades por temor 
al rechazo. No obstante, también se encontró que los y las participantes cuentan con fuentes de apoyo, las cuales les han ayudado en su proceso de afrontar la condición. Por otra parte, todos y todas mostraron ser resilientes ante la situación estresante y utilizan la misma como motor en sus vidas. Al ser jóvenes, se proyectan con un futuro lleno de oportunidades y éxitos, y desde su experiencia pueden apoyar a más jóvenes en su misma situación. Sin embargo, reconocen que hay que educar y generar cambio en el pensamiento colectivo, para que las personas con VIH/SIDA logren vivir en espacios seguros y libres de estigma, donde se respeten sus derechos y dignidad como persona.

$\curvearrowright 2021 \propto$

Díaz Medina, Haylis \& Román Cortijo, Carmen (2021). Cuando quedarse en casa no es una opción: experiencias de las personas sin hogar en el casco urbano de Río Piedras durante la Pandemia por COVID-19: Servicios y movilidad.

Resumen

El propósito de esta investigación fue explorar las experiencias de las personas sin hogar y de los profesionales del Trabajo Social, a través de dos dimensiones: movilidad y servicios. La dimensión de movilidad se integra, porque se reconoce el espacio geográfico como una de las estructuras de mayor contacto en la cotidianidad de las personas sin hogar. De manera, que las decisiones espaciales de la población brindan información valiosa sobre cómo las estructuras sociales y los servicios, les condicionan espacialmente en momentos de emergencia. Mientras, en la dimensión de servicios sociales, se busca conocer cuáles cambios han surgido en los ofrecimientos de servicios para esta comunidad durante la pandemia. La metodología que se utilizó en la investigación fue cualitativa, con un enfoque fenomenológico.

\section{Morales Ramos, Gabriela A. (2021). Retos académicos, sociales y económicos enfrentados por estudiantes de la Universidad de Puerto Rico, Recinto de Río Piedras con la educación a distancia debido al COVID-19.}

Resumen

La pandemia del COVID-19 trajo consigo grandes cambios. Uno de esos cambios fue en el aspecto educativo, ya que se tuvo que cambiar de una educación presencial a una educación a distancia. Esto representó un gran reto para todos los componentes de la comunidad educativa. El marco teórico utilizado en esta investigación fue la teoría de la crisis de Gerald Caplan. Los propósitos específicos de esta investigación fueron: conocer, explorar e identificar los retos académicos, sociales y económicos, que han enfrentado los y las estudiantes de la Universidad de Puerto Rico, Recinto de Río Piedras, con la educación a distancia debido a las medidas que se han tenido que tomar por el COVID-19. En esta

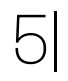


investigación se utilizó un enfoque cuantitativo de tipo exploratorio-descriptivo. El diseño fue no experimental. El instrumento utilizado fue un cuestionario en línea realizado a través de la plataforma Google Forms. El enlace para acceder al cuestionario y la invitación a participar fueron enviados a los y las participantes por correo electrónico, a través, del servicio Cartero RRP. En esta investigación participaron 100 estudiantes de bachillerato, maestría y doctorado. Los hallazgos más importantes de esta investigación fueron los siguientes: los aspectos académicos representaron el reto mayor con la educación a distancia para los y las participantes, seguido por los aspectos sociales y, por último, los aspectos económicos. Las situaciones académicas experimentadas por los y las participantes fueron: el agotamiento físico y mental, la multiplicidad de responsabilidades y la falta de concentración. Las situaciones sociales más señaladas fueron el cambio en las rutinas personales y la falta de interacción con sus compañeros y compañeras de clase. Las situaciones económicas más señaladas fueron: asumir nuevas responsabilidades económicas, la compra de equipo tecnológico y la reducción de ingresos. Los aspectos positivos de la educación a distancia más importantes para estos participantes fueron la reducción en el gasto económico y de tiempo, y la flexibilidad con la que se toman los cursos.

\section{Tanon-Sustache, Militza A. \& Salgado-Velázquez, Heriberto (2021). El accionar de los y las profesionales del Trabajo Social con la violencia de género en Puerto Rico: educación, percepciones y actitudes a mayo del 2021.}

Resumen

En esta investigación se pretendió auscultar la educación, la percepción y la actitud de los profesionales del Trabajo Social sobre el tema de la violencia de género en Puerto Rico, con el propósito de conocer cómo están interviniendo en casos de violencia de género y proponer posibles cursos de acción. Como profesionales del Trabajo Social, tenemos un rol dirigido a defender los derechos humanos que tienen las mujeres, que están afectados por este problema sistemático. La metodología en esta investigación fue una de carácter cuantitativo, dado a que esta nos permite ver los resultados de manera numérica. Seguimos un enfoque basado en la teoría de constructivismo social, la teoría feminista interseccional y de los derechos humanos. El instrumento de recolección de datos fue una escala Likert validada por expertas. Esta investigación se trabajó de manera cibernética debido a la pandemia del COVID-19. Se utilizó Google Forms para realizar el cuestionario, y desde esta misma aplicación, se obtuvieron los resultados para analizarlos. De los hallazgos de la investigación se desprendió, que algunos de los y las profesionales no habían tomado cursos acerca de la violencia de género en su etapa universitaria. La mayoría, un $92.5 \%$, entiende que la violencia doméstica es uno de los mayores problemas sociales del país. Además, un 82.1 por ciento, estuvo de acuerdo en que durante su carrera como profesionales del Trabajo Social atienden casos en los que está involucrada la violencia de género. Cabe destacar que los y las participantes, en su mayoría (85.9\%), estuvieron de 
acuerdo en que las creencias religiosas no influyen en su desempeño, siguiendo así el Código de Ética Profesional por el que se rige esta profesión. Por otra parte, una de las recomendaciones más importantes es que se ofrezcan cursos sobre violencia de género en todas las universidades que otorguen grados en Trabajo Social. Debe incluirse de manera integral las diferentes universidades.

\section{Vélez Vega, Ana L.; Pérez Ortiz, Bianca M. \& Serra Gómez, Indianeth (2021). Percepción de la juventud entre las edades de 15 a 18 años sobre el juvenicidio, las implicaciones en su desarrollo y la importancia del Trabajo Social para esta población.}

Resumen

Esta investigación tuvo como propósito conocer la percepción de los jóvenes de 15 a 18 años, que viven en comunidades con alta incidencia de violencia y juvenicidio en Puerto Rico. Mediante este estudio se pretendió conocer cómo esta percepción y vivencias afectan el tránsito por la etapa de la adolescencia tardía, y los efectos que tienen en el desarrollo físico, cognitivo y conductual de la juventud, al impactar sus próximas etapas de desarrollo y su construcción del mundo, la sociedad y la persona. Los objetivos desarrollados para esta investigación fueron (1) conocer cómo las experiencias vividas de los jóvenes de 15 a 18 años ante el juvenicidio, inciden en su desarrollo social, educativo, cultural y económico; (2) explorar la trascendencia de la intervención del Trabajo Social con jóvenes de 15 a 18 años, que viven en comunidades expuestas al juvenicidio; (3) indagar qué modelos profesionales, de política pública y estrategias de intervención, van dirigidos a visibilizar el fenómeno, al plantear las nuevas propuestas de prevención y acción que presentan los profesionales del Trabajo Social que intervienen con este grupo poblacional. La investigación ofrece un análisis sobre el desarrollo del concepto del juvenicidio. Se problematiza su invisibilización y su perspectiva individual y punitiva. Se exponen los factores psicosociales, la economía, la política, lo cultural y una visión ecológica, para explicar el fenómeno bajo estudio. Para el diseño metodológico se utilizó una metodología cualitativa de tipo exploratorio, a través de entrevistas semiestructuradas individuales. La muestra fue de cinco jóvenes entre las edades de 15 a 18 años y dos profesionales de Trabajo Social, con experiencia directa con la juventud. Los resultados demuestran que el tránsito por la etapa de la adolescencia tardía se ve afectado por la influencia de sus pares, sus familias, y el acceso a recursos. Ayudan en el proceso, una educación de calidad, la participación en actividades culturales, las facilidades recreativas en su comunidad, las expectativas de la sociedad, y la intervención de profesiones relacionados con la conducta humana, como el Trabajo Social. Además, se reconoce la importancia de esta profesión en la intervención con la juventud, para que se les provean servicios de calidad y de prevención primaria. 